\title{
Calculation of Changing of Holding Shares or Currencies by Instant Diffusion Equation of Price Changing with Multiple Sources
}

\author{
Tianquan Yun \\ School of Civil Engineering and Transportation, South China University of Technology, Guangzhou, China \\ Email: cttqyun@scut.edu.cn
}

Received February 20, 2012; revised March 20, 2012; accepted March 28, 2012

\begin{abstract}
The purpose of this paper is application of the instant diffusion equation to the calculation of strategy on changing of owning shares or currencies. The strategy of selling share(s) with maximum changing rate of price-ratio and purchasing share(s) with minimum changing rate of price-ratio (SMaPMi) is calculated by instant diffusion equation with multiple sources of stock-price changing. The spacial coordinate is defined by stock-price changing ratio at a time before diffusion, which ensures that the changing of holding shares using SMaPMi has maximum profit at time next to diffusion ending, based on the comparability of changing rates of stock-price. Summary, SMaPMi would be worked if operation is proper. Calculating example of owning currencies using SMaPMi is given.
\end{abstract}

Keywords: Stock Market Strategy; Strategy of Changing Holding Shares or Currencies; Instant Diffusion Equation of Price Changing

\section{Introduction}

Cyclic changing is a general phenomenon in nature and social life. The so-called "cyclic changing of focus on share(s) with high changing rate from share group to share group" is often seen in Chinese stock markets. According to this phenomenon, a strategy SMaPMi of changing of holding share(s) is designed. There are many strategies used in stock market, for example, the strategy of buying winners and selling losers [1]; cross holding strategy to increase control [2], etc. However, no literature on quantitatively analysis of strategy used in stock market based on certain (not on probability) theory has been found. In this paper, a strategy of selling share(s) with maximum price-ratio changing rate and purchasing share(s) with minimum price-ratio changing rate (simplify as "SMaPMi") is calculated by instant diffusion equation of stock-price changing [3].

In Section 2, the diffusion equation of one source of price changing shown in [3] is repeated and extended to multiple sources of price changing. It also gives more clear explanation on diffusion transmitted each other among sources of price-changing. Further more, the price changing is replaced by the price changing ratio for accurate description, and thus the diffusion equation and its solution of multiple sources of stock-price changing are replaced by its correspondences of stock-price changing ratio.

In Section 3, a strategy of SMaPMi is described.

In Section 4, the spacial coordinate $x$ of stock-price $p(x, t)$ is defined.

In Section 5, discussion on operation in details is made.

In Section 6, a calculating example for changing of currencies using SMaPMi is given. Finally, a conclusion is made.

\section{Instant Diffusion Equation Due to Price Changing of Commodities}

For convenience to the readers, let us repeat the instant diffusion equation shown in [3]:

$$
\frac{\partial p_{j}}{\partial t}=D \frac{\partial^{2} p_{j}}{\partial x^{2}}
$$

where $p_{j}=p_{j}(x, t)$ represents the price of commodity $x$ at time $t$ due to a raising price changing source at $x_{j}$. Equation (1) is explained by the Newton's second law, where $\partial p_{j} / \partial t$ represents a "force" applied in an equilibrium state; $\partial^{2} p_{j} / \partial x_{j}^{2}$ represents "transmitted acceleration"; $D$ is a constants similar to the inertia of mass in mechanic. By method of departing variables, the solution of (1) is shown as follows.

$$
p_{j}(x, t)=A_{j} \exp \left[k_{j}\left(x-x_{j}\right)+c_{j} t\right]
$$


where unknowns $A_{j}, k_{j}$ and $c_{j}$ are determined by initial boundary conditions.. These initial boundary conditions are: 1) $t=0^{-}, p_{j}\left(x_{j}, 0^{-}\right)=p_{j 0}$, by (2), we have

$$
A_{j}=p_{j 0}
$$

2) $\partial p_{j} / \partial t=\left[p_{j}\left(x_{j}, 0^{+}\right)-p_{j}\left(x_{j}, 0^{-}\right)\right] / \Delta t$

$$
=p_{j}\left(x_{j}, 0^{+}\right)-p_{j}\left(x_{j}, 0^{-}\right) / 1=\dot{p}_{j 0}, \text { by (2), we }
$$

have

$$
c_{j}=\dot{p}_{j 0} / p_{j 0}
$$

3) $t=0^{-}, \partial p_{j} / \partial x=\left[p_{j}\left(x, 0^{-}\right)-p_{j}\left(x_{j}, 0^{-}\right)\right] /\left(x-x_{j}\right)=p_{j 0}^{\prime}$, $x \rightarrow x_{j}$, by (2), we have

$$
k_{j}=\dot{p}_{j 0} / p_{j 0}
$$

Substituting (3), (4), and (5) into (2), we have

$$
p_{j}(x, t)=p_{j 0} \exp \left[\left(p_{j 0}^{\prime} / p_{j 0}\right)\left(x-x_{j}\right)+\left(\dot{p}_{j 0} / p_{j 0}\right) t\right](6)
$$

Substituting (2) into (1), we have

$$
D=c_{j} / k_{j}^{2}
$$

If the price changing is falling down, i.e., $c_{j}<0$, then, the last term $+c_{j} t$ in solution (2) is replaced by $-c_{j} t$, such that the constant $D$ is always positive.

The above is repeated from [3]. Now, we make two improvements on [3]:

\subsection{Diffusion Equation with Multiple Sources of Price Changing}

The diffusion equation for one source of price changing is extended to diffusion equation with multiple sources of price changing.

Suppose that there are $n$ sources of price changing appearing consequently at time $t=0^{-}+\theta$, if $\theta \rightarrow 0^{+}$, then, we can consider that the $n(j=1,2, \cdots, n$. $)$ sources of price changing is appearing at the same time interval $\left[0^{-}, 0^{+}\right]$. The diffusion equation due to $n$ sources of price changing is the sum of (1). The solution of diffusion equation due to all $n$ sources of price changing is therefore:

$$
\begin{aligned}
& p(x, t) \\
= & \sum_{j=1}^{n} p_{j 0} \exp \left[\left(p_{j 0}^{\prime} / p_{j 0}\right)\left(x-x_{j}\right)+\left(\dot{p}_{j 0} / p_{j 0}\right) t\right] \\
= & \int p\left(x_{j}, 0\right) \operatorname{ex}\left\{\left[p^{\prime} x_{j}, 0 / p x_{j}, 0\right]\left(x-x_{j}\right)\right. \\
& \left.+\left(\dot{p}\left(x_{j}, 0\right) / p\left(x_{j}, 0\right)\right) t\right\} \mathrm{d} x_{j}
\end{aligned}
$$

where $\int$ replaces $\Sigma$, since $x$ (include $x_{j}$ ) is assumed to be continuous. According to the theorem of middle value of integral, we have

$$
\begin{aligned}
& p(x, t) \\
= & p_{m 0} \exp \left[\left(p_{m 0}^{\prime} / p_{m 0}\right)\left(x-x_{m}\right)+\left(\dot{p}_{m 0} / p_{m 0}\right) t\right](n)
\end{aligned}
$$

where $p_{m 0}=p\left(x_{m}, 0^{-}\right)$is the average stock price of all stocks; $p_{m 0}$ and $\dot{p}_{m 0}$ are its partial derivatives respect to $x$ and $t$ respectively. Equation (9) shows that the solution of multiple sources of price changing can be expressed by one source of price changing at $x=x_{m}$.

It should be noticed that the diffusion process is a process of transmitted each other among all sources and is completed instantly. The diffusion with beginning at time $0^{-}$and end at the time $0^{+}$changes an old equilibrium state to a new equilibrium state. Otherwise, the diffusion process is still continuous, until a new equilibrium state is formed. Since (9) shows that the solution of $n$ sources can be represented by one source at $x=x_{m}$, so that we don't need the details of initial and boundary conditions for each source, but just need the initial and boundary conditions for one source at $x=x_{m}$.

\subsection{Diffusion Equation of Price Changing Ratio with Multiple Sources}

To measure the degree of a changing state, using the rate of price-ratio changing $\partial r(x, t) / \partial t$ is better than using price changing rate $\partial p(x, t) / \partial t$. Where the price-ratio changing $r(x, t)$ is defined by

$$
r(x, t)=\left[p(x, t)-p\left(x, t_{0}\right)\right] / p\left(x, t_{0}\right), t>t_{0},
$$

In the following, we use $r(x, t), r^{\prime}$, and $\dot{r}$ instead of $p(x, t), p^{\prime}$, and $\dot{p}$ respectively for all the above formulas. From (9), we have:

$$
r(x, t)=r_{m 0} \exp \left[\left(r_{m o}^{\prime} / r_{m 0}\right)\left(x-x_{m}\right)+\dot{r}_{m 0} / r_{m 0} t\right](n),
$$

(11) is the solution of diffusion equation of multiple sources of price-ratio changing. Our calculation is based on (11).

\section{Strategy of Selling Shares with Maximum Price Changing Rate and Purchasing Shares with Minimum Price Changing Rate (SMaPMi)}

The strategy SMaPMi is one of strategies used by the author for changing of holding shares. SMaPMi intends to gain maximum difference of stock-price changing from selling and purchasing. The goal of many speculators take part in speculation is just for obtaining different price from purchasing and selling. They do not care much on good or bad of earning ability of company of a share, but just care on how to get maximum profit from trading. So that SMaPMi is a typical strategy of speculation. In the following, the SMaPMi is calculated.

According to the condition of equal price changing of the holding shares, we change the holding shares by

$$
n_{s} p\left(x_{s}, 0\right)\left(1-f_{s}\right)=n_{p} p\left(x_{p}, 0\right)\left(1+f_{p}\right)
$$

where subscripts $S$ and $P$ denote selling and purchasing 
respectively; $n_{S}$ and $n_{P}$ are the numbers of selling and purchasing shares respectively; $f$ is dealing fee per share. $p\left(x_{s}, 0\right)$ is the price of selling shares, which, for SMaPMii, has a maximum changing rate at time $t=0$; while $p\left(x_{p}, 0\right)$ is the price of purchasing shares, which, for SMaPMi, has a minimum changing rate at time $t=0$.

\section{Definition of Spacial Coordinate $x$ of Stock-Price $p(x, t)$}

The spacial coordinate $x$ of price $p(x, t)$ of commodity is defined by the price relation degree among commodities in [3]. Share is a kind of commodity, what is the price relation degree in shares? A stock-price reflects the combination of affecting factors: e.g., earning ability of the company, economic environment (relation between supply and demand; interest rate; exchanging rates; policy; etc.) and the strategy of buyers and sellers, etc. Stockprices connect each other by the connection of companies (e.g., trading connections; guarantee relationship; and loan contract, shares-holding contract, etc.); and the comparability of stock-prices. Comparing to commodity, shares is easier or conveniently to speculate and its price is determined more on the strategy of buyers and sellers.

The key of description of diffusion phenomena of stockprice changing is the definition of spacial coordinate. Deferent definitions lead to different phenomena of diffusion description.

For the diffusion phenomenon of going up (or falling down) of stock-price from a share(s) to other share(s) is called "recycling changing of shares group" in Chinese marketing term, the definition of spacial coordinate is defined by the following process:

According to the market data at time $t_{0}$, all $r\left(t_{0}\right)$ are arranged in order, such that

$$
r_{i}\left(t_{0}\right)=r\left(s_{i}, t_{0}\right)<r_{i+1}\left(t_{0}\right)=r\left(s_{i+1}, t_{0}\right)
$$

Constructing a series:

$$
x_{i}<x_{i+1},
$$

And making a correspondence between these two series, e.g.,

$$
x_{i}=r\left(s_{i}, t_{0}\right)
$$

Then, (15) holds for different symbol of $S_{i}$, i.e., (15) holds for

$$
x_{i}=r\left(x_{i}, t_{0}\right)
$$

Let $x$ be an axis, such that all $x_{i}$ lies on it, then the spacial coordinate $x$ is defined by

$$
x=r\left(x, t_{0}\right)
$$

(16) is the definition of spacial coordinate $x$ of $p(x, t)$ and $r(x, t)$.

\section{Discussion on Details}

At first, we choose a $t_{0}$ or $0^{-}$and a $t$ or $0^{+}$. We can choose any time to be $t_{0}$ and $t$. For example, we choose 2011$12-15$ be $t_{0}$ or $0^{-}$, from the market data, the average price of Shenzhen stock market $p\left(x_{m}, t_{0}\right)=7.53(\mathrm{RMB} /$ share). We choose 2011-12-18 be $t$ or $0^{+}$, then, from the data we get $p\left(x_{m}, 0\right)=7.545(\mathrm{RMB} /$ share $)$, by (10), we have $r_{m 0}=(7.545 / 7.3)-1=0.333$. However, the $r_{m 0}^{\prime}$ and $\dot{r}_{m 0}$ of (11) are not easily to find out, since the average price at $x_{m}$ is not a real share and in fact the stocks is not continuous so that its partial derivatives $r_{m 0}^{\prime}$ and $\dot{r}_{m 0}$ can only be found approximately. Nevertheless we can avoid this difficult by the following calculation by (11):

$$
\frac{r\left(x_{s}, t\right)}{r\left(x_{P}, t\right)}=\exp \left[\frac{x_{s}}{x_{p}}\right]=\exp \left[\left(\frac{r\left(x_{s}, t_{0}\right)}{r\left(x_{p}, t_{0}\right)}\right)\right]
$$

where $x_{s}=r_{s}\left(t_{0}\right)$, the selling share, must be in the pool of holding shares, since in Chinese stock markets the regulation of selling a share must be a holding share. While the purchasing share $x_{p}=r_{p}\left(t_{0}\right)$ may be in or not in the pool of holding shares.

(17) shows that if $x_{S}=x_{\max }$ and $x_{P}=x_{\min }$, then, $r\left(x_{\max }, t\right) / r\left(x_{\min }, t\right)$ becomes maximum. That is, SMaPMi has maximum profit at time $t>0^{+}$, if no new breaking factor on equilibrium state appearing after the end of diffusion $0^{+}$.

Discussion on some details:

1) How to determine the time interval of beginning and end of the diffusion $\left[t_{0}, t\right]$ or $\left[0^{-}, 0\right]$ ?

It depends on the judge, or the wish of the operator. For example, if the operator wishes the price-ratio of the maximum changing rate of the selling share(s) is $10 \%$, then, he or she shall wait the time $t$ until the condition $10 \%$ is reached, i.e.,

$$
r\left(x_{\max }, t\right)=\frac{p\left(x_{\max }, t\right)-p\left(x_{\max }, t_{0}\right)}{p\left(x_{\max }, t_{0}\right)}=10 \%
$$

where $p\left(x_{\max }, t\right)$ and $p\left(x_{\max }, t_{0}\right)$ are known at time $\mathrm{t}$ and $t_{0}$ respectively.

2) Is the time of selling share(s) and purchasing share(s) should be the same?

It depends on the judge of the operator. If the operator can not judge the price of the purchasing share is going up or down, then, it is best to purchase the share at the same time of selling the share with maximum changing rate.

3) How to determine the volumes of selling and purchasing shares?

It depends on the judge of the operator. In the case of lacking in purchasing money, it is best to purchase the volume according to (12). 


\section{Example of Changing of Owning Currencies Using SMaPMi}

The following data are listed from the turnover record of the ICBC account of the author.

(2012-02-16) 2674.44 USD $\rightarrow$ 2050.36 EUR (due to debt crisis of Greek, USD had maximum increasing and EUR had minimum increasing, EUR/USD = 1.3044. According to SMaPMi, sold USD and buy EUR)

(2012-02-20) 2050.36 EUR $\rightarrow$ 2707.09 USD (EUR rebounded but should be temporal, EUR/USD $=1.3203$ )

(2012-03-12) 2707.09 USD $\rightarrow$ 2066.17 EUR (due to better employment record in USA, USD had a maximum increasing and EUR had a minimum increasing, EUR/USD = 1.312). According to SMaPMi, sold USD and buy EUD.

\section{Conclusions}

1) SMaPMi is suited for short term speculation, if operator is proper.

2) The process of diffusion is a process from the be- ginning of a breaking of an old equilibrium state to the end of a new equilibrium state due to inertia. The calculation of strategy of SMaPMi based on diffusion equation of multiple sources, i.e. (17), is suited for time $t \geq 0^{+}$(the end of the new equilibrium state) if no new breaking factor appearing. SMaPMi is also suited for changing of currencies.

\section{REFERENCES}

[1] N. Jegadesh and S. Titman, "Returns to Buying Winners and Selling Losers: Implications for Stock Market Efficiency," Journal of Finance, Vol. 48, No. 1, 1993, pp. 6591. doi:10.1111/j.1540-6261.1993.tb04702.x

[2] R. K. Kakani and T. Joshi, "Cross Holding Strategy to Increase Control: Case of the Tata Group,"XLRT Working Paper, 06-03, 2006, pp. 1-24.

[3] T. Q. Yun, "Instant Diffusion of Price Changing and 'Time-Space Exchange' Description," Technology and Investment, Vol. 2, No. 2, 2011, pp. 124-128. doi: $10.4236 /$ ti.2011.22012 